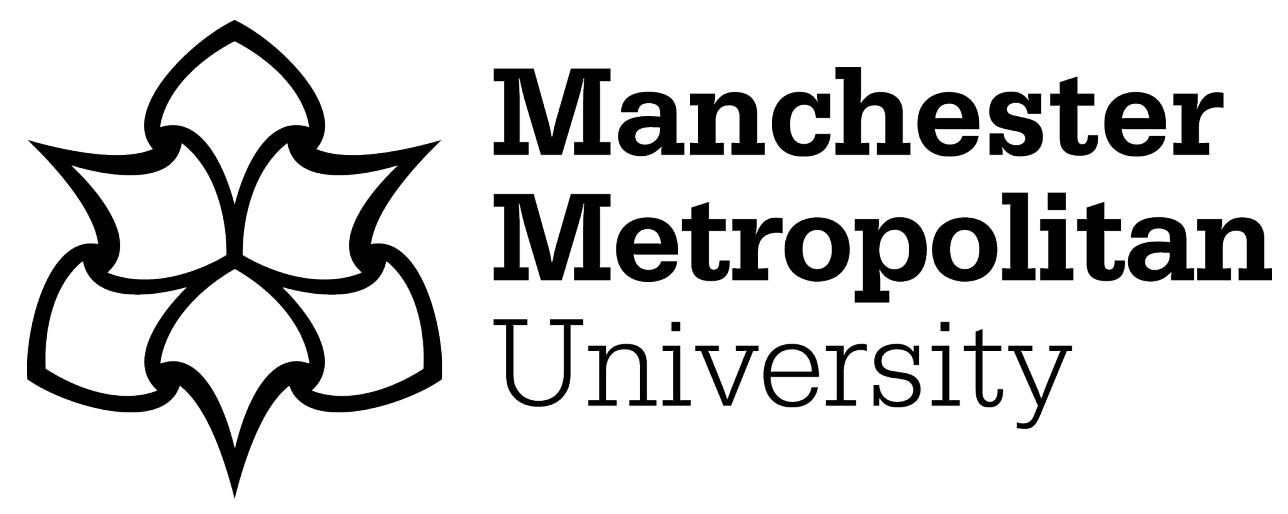

Johnson, F (2018) From Accessibility to Assess-Ability: An Evaluation Heuristic Based on Cognitive Engagement in Search. Lecture Notes in Computer Science, 10766. ISSN 0302-9743

Downloaded from: https://e-space.mmu.ac.uk/620051/

Publisher: Springer Verlag

DOI: https://doi.org/10.1007/978-3-319-78105-1_53

Please cite the published version 


\title{
From accessibility to assess-ability: An evaluation heuristic based on cognitive engagement in search
}

\author{
Frances Johnson ${ }^{[0000-0003-0632-9790]}$ \\ Department of Languages, Information \& Communications, \\ Manchester Metropolitan University \\ Manchester, UK. \\ F.Johnson@mmu.ac.uk
}

\begin{abstract}
This investigation into information searching behaviour focuses on the users' critical assessment of information when found in response to an information need, and on the cognitive aspects of search involving in the user in the assimilation of the information found. The meta analysis of a questionnaire based survey seeks to identify the constructs of the users' assessment of information. Factor analysis of the participants' responses identifies the assessment of the 'cognitive relevance' as vital in distinguishing the searcher who appears to be intent on finding information as opposed to one engaged in the relatively simple task of looking up information. The value in the development of the questionnaire designed to identify the users' cognitive engagement in search is considered for testing the interface designed to optimize the user's involvement in search. Turning to the question of the design of the interface itself, the heuristic of assessability (of the information retrieved) is proposed for use in the expert review of the search interface, and to support the user in their critical assessment and verification of the information relevancy, quality and credibility.
\end{abstract}

Keywords: Search behaviour. Information credibility. Usability.

\section{$1 \quad$ Introduction}

As Wilson et al [1] stated in the introduction to their monograph "From keyword search to exploration: designing future search interfaces for the web", the techniques for retrieving and visualising search results has been well researched and "[are] usually remarkably effective". However they continue to suggest that "recent work has shown that there is substantial room for improving the support provided to users who are exhibiting more exploratory forms of search, including when users may need to learn, discover, and understand novel or complex topics". Current interfaces designed to help the user query, formulate complex search expressions, navigate taxonomies and drill 
down using facets are indeed remarkably effective and the developments that may define potential future user search interfaces, using technologies from gesture to voice to mind control, chatbots and augmented reality look set to be transformative in the way we interact with search systems to find, learn and discover.

In research and development, design and evaluation go hand in hand and with developments in design we need metrics to indicate success. One approach is to set up an information retrieval experiment and measure the performance of search technology in terms of the recall and precision of the search results. However as developments focus on the user, interface and interactivity, approaches to evaluation have developed to take into consideration the complexity of the search processes and to base measures of success in terms of what a successful search might mean to the user. The aim being to design for usability and for the quality of the experience, that is user centered design.

In this investigation into information searching behavior, specifically the users' critical evaluation of information in response to an information need, we aim to identify and characterize the users' involvement in the cognitive aspects of search as they learn about the information sought and assimilate the information found. The searchers' involvement in some critical evaluation of information retrieved is crucial [2] as it is assumed to have a vital role in the assimilation of the information found and in the learning that occurs in the process of searching for information. With regards to an 'anomalous state of knowledge' [3] it is thought that this helps shift the searcher from an uncertain exploratory state to one where the information need is (ideally) clearly defined. The aim of this investigation is to work towards developing a framework for the heuristic based evaluation of the interface designed to optimize users' core activities in search and discovery. This seeks to complement the use of existing and well known heuristics in interface design and metrics to gauge user satisfaction and engagement. The framework proposed is based on key questions the user might ask when searching - such as 'has the retrieval engine worked?', 'is the information retrieved relevant?', 'is the information credible?', 'has my query worked?', 'what have I learnt'? 'what else do I need to know?', and overall, to evaluate the assess-ability of the information retrieved and presented at the interface. This critical approach which leads the user to actively search for information, with intention, assessing both the information retrieved and the interface supporting this activity is arguably a vital literacy necessary for instances of search where the user (with the information need) is bought back into decision making, and for a better experience of searching with current search technologies.

The study on which the new evaluation heuristic is proposed is outlined following a brief background review into past research into users' search behaviour. This is a large body of literature and, as such, is a highly selective look at the cognitive aspects to search, posing the questions: how do we evaluate information found online, what role does this assessment have in the search for information and what are the key influencing factors of the judgments formed? The study itself is questionnaire based and required the participants to respond to a bank of statements relating to their assessment of the information found for given tasks. This involved users making an assessment of the information usefulness that, when made in the dependency state of the searcher and their quest for information, may result in learning and cognitive shifts with respect to searchers' knowledge state. Through factor analysis of the participants' responses, the 
constructs in a possible model of the user's evaluation of information identifies the assessment of the 'cognitive relevance', the judgment of the information in relation to the individual's knowledge and goals, as critical for the development of the proposed interface evaluation of assess-ability.

\section{Interactive Search Behaviour}

The traditional framework for the study of search behaviour depicts the process of search as a series of steps with a goal driven information task. An assessment of relevance would be made to end the search, or to provide feedback in tasks requiring interactivity. The more innovative conceptual frameworks (such as foraging [4]) suggest an unravelling of this process with the view of a planned outcome dismissed in favor of an evolving and adaptive process. Here the path is not a straight trajectory but a process of learning and thus an evolving search. The searcher gathers and interacts with the information creating a personal perspective and learning as the search progresses. This shift in the view of search is conceptualized in models that recognize a user's anomalous state of knowledge and interactions with the information encountered. Toms [5] explains search has become more an 'immersion in a body of information'.

The shift in the concept of search can also be suggested in the development of the search interface. The relationship of the user, information and search as discussed in [6] and with regards to early interface designs, reviewed in [7] focus on the searcher in learning how to search or, more precisely, learning how to query on the index. Experimental visual interfaces for example, that show the impact of formulating the query with Boolean operations, or the visualization of the frequency of the query terms in the retrieved results, arguably invite the searcher to think about and learn how best to formulate the query to match against the system's index. Furthermore user studies of the searcher, in formulating the query and controlling the search as an effective series of moves (e.g., in [8]), are by and large based on an assumption that the searcher has a good idea of the target information sought and is learning how to best ask for the information. In comparison, the modern search interface, with for example visuals of clusters of extracted terms, appear better suited to the conceptualisation of search as exploration as the searcher evaluates the information retrieved and assimilates this information with respect to their current knowledge state. Supporting the users' exploration the interface design ultimately is for the searcher to experience 'flow' [7] again suggesting a subtle shift in the concept of search in the interface design supporting the searcher in learning how to search, for example how to submit the query, to inviting the searcher to learn from the information retrieved so as to continue the query. In this view of search as learning, the users' critical evaluation of information has a vital role.

An effective retrieval system is one which performs, both in terms of providing and facilitating access. The interest in the user and their interactions shifts the evaluation from that of system performance to questions on and around how well the interface supports the user in this task-directed goal of finding information. That is, to ask the questions, how usable and how useful are the interface features and their design? According to the definition of usability provided by the ISO standard $(9241,1994)$ we 
should be designing the system with respect to "the effectiveness, efficiency, and satisfaction with which specified users achieve specified goals" and further assess usability as a "measure of the quality of a user's experience when interacting with a service or resource" [9]. To obtain a user evaluation we can measure "task success on finding items on a subject, along with other measures such as the user's perception of satisfaction. Usability heuristics may also be deployed both in the design and in the evaluation of the interface optimized to assist users in accomplishing the task. Thus evaluation metrics have an important role in user centered design. For example, [10] found significant effect of visually appealing interfaces on performance including completion time, and that there is positive correlation between the interface design and interest levels when searching for complex search tasks or self-chosen tasks [11]. Central to Human Computer Interaction is the maxim that the interface itself should disappear from the user's focus so that they concentrate only on the task in hand. For this, arguably design needs to go beyond usability assessment and evaluate the enabling of the search process taking into consideration the complexity of the users' interactions, both behavioural and cognitive. That is, the evaluation of the user experience demands an understanding of the search processes. One in which the user endeavors to- find the query terms, formulate search expressions, -refine the search based on feedback, -, spot relevant items, - decide what to do next - compare information retrieved and, - attempt to ensure that relevant information is not overlooked. Once we begin to think about what a successful search might mean to the user we can identify the design (and the evaluation) to support user-information interaction in search.

\subsection{Research Questions}

This study investigates users' interactions with information when involved in its critical evaluation conducted in a dependency context between user and the information. Broadly speaking the questions posed are, what do we think when engaged in the critical evaluation of the information found online, on what criteria do we assess the information and what might be the factors influencing the judgments formed? Thus, following Toms [5], the intention is to focus on the cognitive activity of search. The core criteria in evaluating information retrieved in a search context is relevance, an assessment of the topic match between the query and the information retrieved. Previous researchers have distinguished 'topical' relevance from 'situational' relevance that relates to the perceived utility of the document to the users' real situation or task in hand [12] and that may have a prerequisite judgment of 'cognitive' relevance, that the document is understandable and informative given the users' current state of knowledge. It is therefore assumed that formation of cognitive relevance judgments are critical activities in conducting an effective and dynamic search. The study, asks how searchers, in evaluating the information, form a personal perspective on the information and the factors that can influence this judgment. This insight is sought to inform interface design perceived to be supportive of the users' cognitive behaviour in carrying out the search. 


\section{Factoring the Constructs of Relevance Judgments}

The aim of the study was to obtain a characterisation of participants' interactions with the retrieved results from two assigned search tasks. The participants were 102 students from Information related courses at a UK university. The task to find information was set as an exercise to be completed for the following week's class and participants were asked to complete the questionnaire following completion of each search. Using Google, participants were asked a 'general' task to find information on Alan Turing and his contribution to the development of Computing. The expectation being that one or two encyclopaedic type of articles would satisfy this information quest. Whilst the query put to Google Scholar was more open ended to find backgrounder information on the topic of young people's use of Short Message Services (SMS) and its effect on their written language. The expectation being that the participants would have to learn a bit about this topic and find several sources to satisfy the information quest.

A questionnaire was designed to collect data on the assessments made with regards to the relevance of the information found and the assessment of the search engine used. The questions (items) were drawn from previous research which identifies and describes the judgements people make when assessing information retrieved as relevant (as detailed in [13]). The (meta) analysis here is to highlight the differences in the assessments made (of the retrieved information) and in the perceptions formed (of the search engines) when the participants' task was a straightforward 'look up' task and one where there was some simulation of a gap in knowledge and the need to look up and learn about the topic. The comparison thus described was between the 'look up' task using Google and on the 'research task' on Google Scholar (GS) and aimed to provide a characterisation of the cognitive processes when involved in search.

The Likert-style statements (items) aimed to reflect the constructs of information evaluation in the search context. Previous research identifies three types of relevance judgements, topical, cognitive and situational and these were drawn upon to suggest their assessment of the information retrieved. From these a series of statements were drawn up to include in the questionnaire and to avoid asking the participants to respond directly about their assessment of the relevance of the retrieved results. As follows:

- Topical relevance, a judgment of information 'aboutness' and the relation to the query topic is fundamental to search and participants were asked to give their level of agreement to statements such as, the information is related to the topic of interest

- Cognitive relevance refers to some judgment of the informativeness with respect to the user's state of knowledge and, in this personal perspective there is some assimilation of the information. Participants were asked to consider the information retrieved and respond to statements such as, the information has helped me to learn about the subject as a possible indirect measure of cognitive relevance.

- The assumption is that these judgments are formed as a prerequisite for the application of the information to resolve the problem. The two stages of information interaction is clear in Blandford \& Attfield's [2] definition where first the information is acquired and then applied to the situation. In this study the participants were required to find information only therefore only a measure of confidence that the information 
would be useful was sought in responding to items, such as if I knew someone was looking for this information, I would tell them about this.

To investigate the influence of the factors that may be used in making relevance judgments, including document style, recentness, novelty and scope, the questionnaire included 4-5 items relating to these and which as such may further distinguish the users' assessment of the information retrieved, such as the information is accurate, I can believe the content, the information appears authoritative, the information is well written. With respect to the interactive context, and the user assessment of the interface two further constructs were considered as having key importance. Firstly, information interaction may be viewed as a dialogue between the user and the information retrieved with the interface facilitating the cognitive processing involved. It is possible then, if not likely, that the perceived ease with which the user can form the relevance judgments will affect and influence the judgment itself. Thus, a small number of items were included to measure this construct, such as, it was easy to identify what the information was about and I felt that my query had been understood. A further group of items, such as the system is good at finding information that matches my query were included to measure the perceived system effectiveness - that is, 'does it work?' The questionnaire was thus developed to manifest the participants' cognitive processing in search through their responses to this bank of items based on the constructs of topic relevance, cognitive relevance, situational relevance/confident use, information content, style and scope, and on the perceived support and effectiveness of the interface and system. The questionnaire was developed as a psychometric tool to investigate the core factors in users' assessment of the information and of the system in the interactive search.

\subsection{Exploratory Factors}

The statistical procedure of factor analysis was used to extract intended constructs when measured with the multiple statements. If the items for a construct are well designed, they should converge and form a major factor [14]. The Kaiser-Meyer-Olkin (KMO) values were greater than the recommended value 0.6 indicating sampling adequacy of the data prior to conducting factor analysis. Cronbach's alpha coefficient confirmed the reliability of the data in terms of internal consistency. These ranged from 0.706 to 0.926 , which is higher than the minimum cut-off of 0.7 . Table 1 reports the principal component analysis results with varimax rotation using SPSS. Two separate analyses were conducted on each of the datasets (the Google task and the Google Scholar (GS) task) in turn. An alignment across the factors for the Google and GS tasks was sought with a slight difference in the labelling of the factors, and the actual differences in the composition of these comparable factors are as follows. When asked to think about using the information retrieved using Google (query: Alan Turing), all of the items in the factor $[1 \mathrm{G}]$ 'Content Credible' also appeared in the factor [1GS] 'Content Credible and Relevant' formed when thinking about the information retrieved using Google Scholar (for the query topic: use of Short Message Services). These were I can believe the content, [...] is accurate and appears to be authoritative. The factor [1GS] additionally included the statements, the content is totally related to the topic of interest, is 
very easy for me to understand, is well written and tells me most of what I need to know. These additional items might indicate that the participants were assessing the relevance of the information retrieved, as well as the credibility of the information retrieved. The sense that the participants were assessing the relevance of the information retrieved when searching for the task on Google Scholar is further borne out in the different items distinguishing the factor [2G] and factor [2GS], labelled as 'Expected Relevant' and 'Cognitive Relevant' respectively. These factors had in common the items The information found seems to be the right amount for me, is a sufficient to complete the assignment, and I expect to feel well informed on this topic. Factor [2GS] additionally included the items, I expect this information to be very easy to read, easy to understand, I would use this information and [it] has helped me to learn about the subject. Factor $[2 \mathrm{G}]$ on the other hand additionally included only the item I expect to know more about the topic once I have read this information. Further indication that the participants were involved in assessing the information retrieved when using Google Scholar is in the items forming the factors [3GS] labelled 'Confident Useful' when compared to the factor $[3 \mathrm{G}]$ in the Google task. In common were the statements, If asked, I think that other people would value this information, and If I knew someone was looking for this information, I would tell them about this. Whereas factor [3GS] contained the items, this information would help me to complete the assignment, and I expect to know more about the topic once I have read this information whilst factor [3G] included the items I expect this info to be very easy to read and I would use this information to work on the task in hand. The factors formed represent the users' assessment of the information as credible, relevant and useful with common items found in both data sets. The major differences, however, relate to the assessment of relevance in the GS task. Of particular note is the inclusion in Factor [2GS] of the item which mentions learning, 'this information has helped me to learn about the subject' in assessing relevance. It is further noted that the highest loading item in Factor [3GS] labelled 'Confident Useful' refers to the value of the information whereas in $[3 \mathrm{G}]$ instead the highest item refers to ease of understanding. This may suggest that 'ease of reading/use' influences the user's judgment of usefulness in the 'look up' context, whereas in the 'research task' the assessment of information usefulness is made with greater respect to the task context or situation.

Table 1. Factors in the evaluation of the information retrieved

\begin{tabular}{|c|c|c|c|c|c|}
\hline \multicolumn{3}{|c|}{ Google Scholar Factors } & \multicolumn{3}{|c|}{ Google Factors } \\
\hline & Item (abbreviated) & & & Item (abbreviated) & \\
\hline $1 \mathrm{GS}$ & $\begin{array}{l}\text {... content is accurate } \\
\text {...related to topic of interest } \\
\text {...easy for me to understand } \\
\ldots \text { believe the content } \\
\ldots \text { appears authoritative } \\
\ldots \text { is well written } \\
\text {... tells me most of what I need }\end{array}$ & $\begin{array}{l}.831 \\
.765 \\
.753 \\
.747 \\
.658 \\
.652 \\
.613\end{array}$ & $1 \mathrm{G}$ & $\begin{array}{l}\ldots \text { believe the content } \\
\ldots \text { appears authoritative } \\
\ldots \text { of good quality } \\
\ldots \text { content is accurate }\end{array}$ & $\begin{array}{l}.850 \\
.847 \\
.815 \\
.717\end{array}$ \\
\hline $2 \mathrm{GS}$ & $\begin{array}{l}\ldots \text { the right amount for me } \\
\ldots \text { is sufficient } \\
\text {... will be easy to read } \\
\text {... will find easy to understand }\end{array}$ & $\begin{array}{l}.855 \\
.757 \\
.756 \\
.731\end{array}$ & $2 \mathrm{G}$ & $\begin{array}{l}\text {.. is sufficient } \\
\ldots \text { I expect to know more once read } \\
\ldots \text {... feel well informed }\end{array}$ & $\begin{array}{l}.834 \\
.769 \\
.734\end{array}$ \\
\hline
\end{tabular}




\begin{tabular}{|c|c|c|c|c|c|}
\hline & $\begin{array}{l}\text {...I will use this information } \\
\text {...has helped me to learn } \\
\text {... feel well informed }\end{array}$ & $\begin{array}{l}.666 \\
.656 \\
.637 \\
\end{array}$ & & $\begin{array}{l}\ldots \text { helps me complete the assignment } \\
\ldots \text { the right amount for me }\end{array}$ & .631 \\
\hline $3 \mathrm{GS}$ & $\begin{array}{l}\text {...other people would value this } \\
\text {...if looking I would tell some- } \\
\text { one about this } \\
\text {...helps me complete the assign- } \\
\text { ment } \\
\text {... I expect to know once read }\end{array}$ & $\begin{array}{l}.854 \\
.834 \\
.675 \\
.606\end{array}$ & $3 \mathrm{G}$ & $\begin{array}{l}\text {... will find easy to understand } \\
\text {...if looking I would tell someone } \\
\text { about this } \\
\text {... will be easy to read } \\
\text {... w will use this information } \\
\text {... other people would value this }\end{array}$ & $\begin{array}{l}.810 \\
.794 \\
.743 \\
.734 \\
.665\end{array}$ \\
\hline
\end{tabular}

The questionnaire also asked the participants to respond to a set of items relating to the assessment of the system. These either related to the perception that the system worked (Factor5), the system works well in suggesting information, is good at finding information for my query and I can use to get the best results or with respect to the support the participants felt they experienced (Factor 6), my query had been understood, it was easy to identify what the information was about and easy to see why retrieved. There was little variation found in these factors when assessing Google and when assessing Google Scholar. However running multiple regressions on this data does show these factors account for about half of the variance (45\%) in factor [2GS] Cognitive Relevant; but, only $29 \%$ of the variance, in $[2 \mathrm{G}]$ Expected Relevant judgment on Google. Factor 5 relating to user perception of system effectiveness also held a moderate association at $p<.001(\beta=.457)$ with factor [2GS] and again a weaker association with factor [2G] at $\mathrm{p}=.007(\beta=.331)$.

\section{Discussion: Assess-ability as a New Evaluation Heuristic}

With regards to the premise that search involves learning in the context of a gap in knowledge, this study explores the user's critical assessment of the information found. In the analysis of the questionnaire relating to the users' assessment, this study provides some evidence that the participants formed slightly different judgments according to the task. Assessment of relevance appeared to be more embedded in the evaluation of the GS task with the user engaged in assessing 'cognitive relevance' assimilating the information found. In the 'look up' task on Google assessment of the information appears to be less critical based on an expectation of topic relevancy. That is, the evidence presented here tentatively points to a difference in behaviour, especially cognitive, when searching with intention to find information (when compared to looking up information). The limitations of the study are, however, acknowledged and further investigation is recommended with the deployment of the psychometric questionnaire to further research the user's critical assessment of information found in search contexts. Further insight could be usefully gathered in user studies which involve the participants searching for different types of tasks for example with 'real' information needs, and in differing professional contexts. Further validation of this data collection instrument may be sought in correlating the searchers' critical assessment of information with some further measure of satisfaction or engagement. A searcher who appears to be involved in assessing cognitive relevance and assesses the search engine as supportive may report feeling more engaged in exploring new or complex topics. Developments 
in the design of the interface to enhance the user experience may also be gauged using the questionnaire to collect data on their interaction with the information found.

Furthermore, identifying the core activity of assessing cognitive relevance may allow design to focus in the property of the 'assess-ability' of the interface to support the user in their critical assessment of the information retrieved. Nielsen's usability heuristics are de facto in user experience design comprising the ten usability principles which if adhered to can help ensure that the interface uses the users' language, helps user recognize rather than recall, gives consistent feedback on the system status and generally instil a confidence in using the interface. The development of the principle of assess-ability, as a heuristic, would question the support given to assist the user in assessing the information retrieved and help design to support this vital component of search behaviour. The types of questions that may be posed in developing the assessability heuristics may be drawn from recent studies that reveal the features that people claim to use when assessing information. The $\mathrm{C} 3$ (Content Credibility Corpus) [15] containing 15,750 evaluations of 5543 Webpages by 2041 participants, for example includes over 7071 annotated textual justifications of credibility evaluations of over 1361 Webpages. Analysis of these comments involved labelling the factors mentioned as influencing credibility assessment and were grouped into six categories. Each of these represent questions that someone might ask when assessing credibility, as: What kind of Web page is it? $\bullet$ Is the content of commercial character? $\bullet$ Who is the author or publisher? $\bullet$ How is the Web page designed? $\bullet$ Is the textual content of high quality? - Is the information on the Web page verifiable? The extent to which the presentation of the information retrieved enables the user to ask and answer some or all of these questions could form the basis of the assess-ability heuristics. For example, to ask: Is the user able to assess who is the author or publisher? Can the user determine what kind of web page/ the type of article? Can the user assess the quality of the information, for example in assessing the metadata of date and the article references? The presentation of this information may make use of devices to separate out types of content, for example adverts on the page, or thumbnail to signal the type of page, and novel visuals such as 'Wikitrust' [16] designed to show editorial control and help the user to see which parts of the article are credible. The premise being that the more the user is able to assess the information retrieved, the more they will engage with the information, as is vital to searching for information. It is less obvious, however, how to provide support in asking the questions that rely more on the semantic features of the source, i.e., its relevance, completeness, scope and neutrality [17]. Asking the question - 'Can the user verify the information?' requires the user to interact with the information on a deeper level and to check accuracy and completeness and, in search contexts, to relate the information found to one's prior knowledge. In identifying the factors influencing the users' verification of information Brand-Gruwel [18] found that the strategies used involved the user in checking consistency with other sources, making connections to previous knowledge and interestingly, a verification strategy of 'trying to discern author's motive'. The design to assist the user in making this assessment is challenging but may be partly achieved by enabling the searcher to look up the author (if known) and to assess credibility markers of the author's transparency related to their motive in providing the information. Links may be followed enabling the searcher to verify content and, 
critically design tactics (such as the highlighting query terms in retrieved results) may be used to encourage assessment with regards to the users' information need. Here the assess-ability of the information refers to a less tangible property with its relevancy drawing on user's personal knowledge and information requirements. The value of the assess-ability heuristic is therefore not to determine design but rather to draw attention to the impact of design and features that the user perceives as supporting their interaction when assessing the credibility, relevance and usefulness of retrieved information.

\section{Conclusion}

A novel approach to investigating the users' cognitive activity in the critical assessment of information is presented here, and analysis suggests the constructs of the users' judgment. Interestingly, a distinct evaluation could be discerned in which the users appear to form a personal perspective on the information, critically evaluating the information in respect to their goal to find information. As a core and vital aspect of search activity, the study on assessing information provides a framework for thinking about how the information may be presented at the interface and designed for assess-ability. The framework of the heuristic based evaluation of assess-ability, supporting assessment of credibility and relevance, whilst in an early stage of development, provides an approach for interface design to support and evaluate the user's cognitive engagement in search.

\section{References}

1. Wilson, M.L., Kules,B., Schraefel, M.C., Shneiderman, B. From keyword search to exploration. In: Wilson, M.L et al. (eds.) Foundations and Trends in Web Science pp. 1-97 (2010)

2. Blandford, A., Attfield, S. Interaction with Information. Morgan \& Claypool, CA. (2010)

3. Belkin, N.J., Cool, C., Stein, A., Theil, U. Cases, scripts and information seeking strategies: on the design of interactive irs. Expert Systems with Applications. 9, 379-395. (1995)

4. Pirolli,P.,Card, S.K. Information foraging in information access environments. In: Proc. of the Conf. on Human Factors in Computing Systems, ACM New York, pp.51-58. (1995)

5. Toms, E. Information interaction providing a framework for information architecture. Journal of the American Society for Information Science, 53(10), 855-862. (2002)

6. Johnson, F.C. Shifting Contexts: relating the user, search and system In:Efthimiadis EN et al. (Eds) Teaching and learning in information retrieval,.Springer,Heidelberg (2011).

7. Hearst, M.A. Search user interfaces. Cambridge University Press, (2009).

8. Wilson, M.L., Schraefel, M.C.,White, R.W. Evaluating advanced search interfaces using established information-seeking models. Jasist , 60(7) 1407-1422. (2009)

9. Nielsen,J. Usability 101:Introduction to usability https://www.nngroup.com/ articles/usability-101-introduction-to-usability/[17/09/2017]

10. Moshagen,M., Thielsch, M. T. Facets of visual aesthetics, Int. J. of Human Computer Studies, 68(10) (2010).

11. Arapakis, I., Jose, J.M., Gray, P.D., Affective Feedback: An Investigation into the Role of Emotions in the Information Seeking Process, ACM SIGIG, pp. 395 - 402 (2008)

12. Saracevic, T. Relevance reconsidered. In: Proceedings of the conceptions of library and information science (CoLIS 2), ACM Press, New York, pp.201-218 (1996)

13. Johnson, F., Rowley, J., Sbaffi, L. Information Interactions in the Context of Google. Journal of the Association for Information Science \& Technology, 67(4), 824-840 (2016) 
14. Hair, J.F., Tatham, R.L., Anderson, R.E., Black W. Multivariate Data Analysis. 5th Ed. Prentice Hall, NJ. (1998).

15. Kakol, M., Nielek, R., Wierzbicki, A. Understanding Web content credibility using the Content Credibility Corpus Information Processing \& Management 53, 1043-1061, (2017)

16. Wiki-Watch: evaluate the quality of Wikipedia's articles. http://blog.wiki-watch.de/?p=497

17. Lucassen, T., Schraagen, J.M. The influence of source cues and topic familiarity on credibility evaluation. Computers in Human Behaviour, 29(4), 1387-1392 (2013)

18. Brand-Gruwel, S., Kammerer, Y., van Meeuwen, L., Source evaluation of domain experts and novices during Web search. Journal of Computer Assisted Learning.11, (2017). 